\title{
Synthesis of Titanium Containing SBA-15 and Its Application for Photocatalytic Degradation of Phenol
}

\author{
Lili Yang, ${ }^{1}$ Zeyu Jiang, ${ }^{1}$ Sufeng Lai, ${ }^{1}$ Chongwen Jiang, ${ }^{1,2}$ and Hong Zhong1,2 \\ ${ }^{1}$ Department of Chemical Engineering, College of Chemistry and Chemical Engineering, Central South University, \\ Changsha 410083, China \\ ${ }^{2}$ Key Laboratory of Resource Chemistry of Nonferrous Metals, Ministry of Education, Central South University, \\ Changsha 410083, China
}

Correspondence should be addressed to Chongwen Jiang; jcwcsu@csu.edu.cn

Received 13 May 2014; Revised 29 August 2014; Accepted 30 August 2014; Published 9 September 2014

Academic Editor: Deepak Kunzru

Copyright (C) 2014 Lili Yang et al. This is an open access article distributed under the Creative Commons Attribution License, which permits unrestricted use, distribution, and reproduction in any medium, provided the original work is properly cited.

We synthesized Ti-containing SBA-15 by one-pot hydrothermal method and extensively investigated its physicochemical properties and examined its photocatalytic activity to degrade phenol. It's shown that appropriate amount of titanium could be effectively incorporated into the framework of SBA-15 without provoking structure change and the tetrahedrally coordinated titanium species were well-ordered in Ti-incorporated SBA-15. Under UV-irradiation, the Ti-containing SBA-15 exhibits good photocatalytic activity for phenol degradation in the simulated waste water. The higher photocatalytic activity of Ti-containing SBA-15 to degrade phenol was ascribed to the more titanium content and anatase titanium dioxide. In addition, the new photocatalyst displays a good regeneration and cyclic performance after six runs.

\section{Introduction}

Due to their environmental toxicity, persistency, ubiquitous presence and carcinogenic, phenols and their derivatives in drinking water are harmful to human health and ecosystems [1]. The increasing pollution of phenols and other associated environmental hazards in waste water has become a serious issue. As a result, the development of methods to remove the contaminants from waste water has gained considerable interest, and a variety of practical water purification processes to remove phenol pollutants have been proposed including chlorination, solvent extraction, activated carbon adsorption, microbial degradation and membrane separation. However, these techniques are subjected to either complicated procedures, expensive cost, or producing hazardous by-products [2]. In recent years, the heterogeneous photocatalytic oxidation process has emerged with a good potential through which the complete mineralization of highly toxic phenol compounds in water can be achieved [3].

Titania, especially anatase phase, has been proven to be one of the most efficient photocatalysts due to its appropriate electronic band structure, photo-stability, chemical inertness, and commercial availability. Considering the mechanism of a heterogeneous photocatalytic reaction, it has been known that the degradation rate of the reactant using $\mathrm{TiO}_{2}$ in heterogeneous photocatalysis largely depends on the adsorption capacity which is associated with the specific surface area. A great number of researches have been carried out to improve the photocatalytic activity of $\mathrm{TiO}_{2}$ by using a solid support with a primary objective to increase the amount of active sites per unit area, and achieve a higher photocatalytic reaction rate. On account of this, several investigators have chosen to incorporate $\mathrm{TiO}_{2}$ in supports such as silica [4], alumina [5], zeolite [6], and activated carbon [7], and so forth to enhance the efficiency, mechanical, and thermal stability. Even though most of the mixed materials show good efficiency, SBA-15 has been regarded as a promising support, which facilitates adsorption and contributes to the more loading of active sites due to its high surface area, large pore volume, tunable pore size, thick pore wall, good stability, and good performance as effective adsorbents since it has been discovered [8]. Moreover, the addition of $\mathrm{SiO}_{2}$ phase 
to $\mathrm{TiO}_{2}$ facilitates in the effective dispersion of the anatase crystallites [9] that serve as photoactive centers essential for the cleavage of organic pollutants and their degradation to innocuous intermediates and by-products [10]. Jung et al. [11] prepared titanium containing SBA-15 via conventional hydrothermal method and found that its photocatalytic activity to decompose the orange II increased with increasing of Ti content. Zhang et al. [12] synthesized a series of Ti-SBA15 in the self-generated acidic environment exhibiting much higher photodegradation ability towards Rhb than pure $\mathrm{TiO}_{2}$. In spite of the successful application of SBA-15 supported catalysts in various catalytic processes, there is no report to degrade phenols with $\mathrm{TiO}_{2} / \mathrm{SBA}-15$ mesoporous catalysts under UV irradiation.

Recently, many efforts have been made to prepare Ticontaining SBA-15 through post-synthetic grafting procedures or direct synthesis $[12,13]$. The post-synthetic method usually forms the metal oxides in the channels or external surfaces of catalysts, which would lead to blocking the channels and not allowing the easy access of reactant molecules to the reaction sites in the porous matrix. Comparatively, the direct synthesis can avoid the partial pore blockage and provide well distribution of species in the framework and surface without any decrease of mesopore size. Moreover, direct synthesis is simpler and energy saving. In our present work, a onestep process to prepare the Ti-containing SBA-15 catalyst was developed to solve the problems in two-step synthetic method such as the formation of metal oxide catalysts on the surface, blocking the pore of support, partial breakage of the wall and decrease in surface area. Besides, the ethanol co-solvent was used to dissolve tetrabutyl titanate to match the hydrolysis rate with that of silicon source, leading to effective introduction of $\mathrm{Ti}$ into the silica framework. The synthesized materials were characterized by powder X-ray diffraction (XRD), $\mathrm{N}_{2}$ adsorption-desorption, FT-IR, UV and $\mathrm{X}$-ray photoelectron spectroscopy (XPS), respectively. Their catalytic performance towards the photodegration of phenol was evaluated. Furthermore, the Ti-SBA-15 used as the solid heterogeneous catalyst in this study can be easily recovered and reused without obvious loss of activity.

\section{Experimental}

2.1. Materials. Tetraethylorthosilicate (TEOS) and triblockcopolymer (poly(ethylene glycol)-poly(propylene glycol)block-poly(ethylene glycol)) of molecular weight 5800 (Pluronic P123) purchased from Aldrich were used as silicon source and structure directing agent, respectively. All other chemicals were used as received.

2.2. Preparation Method of One-Pot Synthesized Ti-SBA15. Ti-substituted SBA-15 materials were synthesized by hydrothermal method using TEOS and tetrabutyl titanate (TBT) as the sources of $\mathrm{Si}$ and $\mathrm{Ti}$, respectively. In a typical synthesis, $4.0 \mathrm{~g}$ of pluronic P123 was dissolved in $146.67 \mathrm{~g}$ of distilled water and $6.7 \mathrm{~mL}$ hydrochloric acid (37 wt.\%) under stirring at room temperature for $1.5 \mathrm{~h}$. Thereafter, $8.72 \mathrm{~g}$ of TEOS was dripped into the solution and stirred for $2 \mathrm{~h}$. Then the required amount of TBT in $10 \mathrm{~mL}$ ethanol was slowly added to the acid solution under vigorous stirring at $38^{\circ} \mathrm{C}$. After stirring for $20 \mathrm{~h}$, the resultant mixture was transferred into a polypropylene bottle and aged at $100^{\circ} \mathrm{C}$ for another $20 \mathrm{~h}$ without stirring. The resulting mixtures were filtered, washed, and dried at $100^{\circ} \mathrm{C}$ overnight. After calcination at $550^{\circ} \mathrm{C}$ for $5 \mathrm{~h}$ in air, the mesoporous products were obtained denoted as Ti-SBA-15.

2.3. Methods of Characterization. XRD patterns of the samples were recorded on a Rigaku D/Max-2500/pc powder diffraction system using $\mathrm{Cu} \mathrm{KD}$ radiation $(40 \mathrm{kV}$ and $250 \mathrm{~mA}$ ) over the range $0.5^{\circ} \leq 2 \theta \leq 5^{\circ}$ (low angle) and $10^{\circ} \leq$ $2 \theta \leq 80^{\circ}$ (high angle). Diffuse reflectance UV-vis spectra was recorded in the range of $190-800 \mathrm{~nm}$ at room temperature by a Shimadzu UV-2450A double-beam digital spectrophotometer with $\mathrm{BaSO}_{4}$ as reference. FT-IR spectra of the samples were measured using an AVATAR-360 spectrometer. Nitrogen adsorption-desorption isotherms were obtained using a Micromeritics ASAP 2020 Gas Adsorption Analyzer at $77 \mathrm{~K}$ after the mesoporous materials were degassed in vacuum at $200^{\circ} \mathrm{C}$ for $5 \mathrm{~h}$. Surface area and total pore volume were determined by using the Brunauer-Emmett-Teller (BET) equation and the Barrett-Joyner-Halenda (BJH) method, respectively. The pore size distribution was calculated from the analysis of the adsorption branch of the nitrogen isotherm using the BJH method. XPS analysis was performed on ESCALAB 250Xi type of X ray photoelectron spectrometer produced by ThermoFisher-VG Scientific.

2.4. Photocatalytic Properties of the Samples. To evaluate the photocatalytic properties of the samples, photocatalytic reaction experiment was performed in a batch of glass reactor. $200 \mathrm{~mL}$ phenol solution $(100 \mathrm{mg} / \mathrm{L})$ as a model compound was added to the glass reactor in the presence of hydrogen peroxide and Ti-SBA-15 $(0.2 \mathrm{~g})$. The illumination was provided by a $250 \mathrm{~W}$ medium pressure mercury lamp $(365 \mathrm{~nm})$. The adsorption equilibrium experiment was carried out before illumination in order to avoid the effect of the surface adsorption. To determine the concentrations of phenol solution during the process, $5 \mathrm{~mL}$ of the solution from the reaction mixture was taken, subsequently centrifuged, and filtered through a millipore filter to obtain the filtrate for concentration determination by using 4-Aminoantipyrine Spectrophotometric Method. The phenol concentration was calculated from the absorbance at $510 \mathrm{~nm}$ using a calibration curve.

\section{Results and Discussion}

3.1. Characterization of Prepared Samples. The low angle XRD patterns of the parent SBA-15 and the representative Ti-SBA-15 sample are shown in Figure 1(a). Both of the samples exhibit very similar patterns where three wellresolved diffraction peaks corresponding to reflections of (100), (110), and (200) planes of a 2D hexagonal structure [14]. However, a slightly lower intensity of the peak was observed for Ti-SBA-15, showing the decrease crystallinity due to the 
incorporation of heteroatoms into the framework of SBA- 15 [12]. This confirmed that the introduction of titanium did not affect the formation of the hexagonal pore structure of SBA15. The high angle XRD patterns are also shown in Figure 1(b) (inset). The high angle XRD results clearly indicate that some amount of Titanium can be incorporated into the silicate framework of SBA-15 under the experimental conditions [13]. The materials with higher Si/Ti ratio in the structure only show a broad signal between $15^{\circ}$ to $25^{\circ}$ due to amorphous silica. However, the specific peaks at $2 \theta=25.3^{\circ}, 37.8^{\circ}, 55^{\circ}, 63.2^{\circ}$ in Ti-SBA-15 sample with a Si/Ti ratio of 10 were ascribed to the anatas phase, and no reflection of rutile phase was detected. These are consistent with results in the previous work by Cani and Pescarmona [15]. Generally, it is believed that the absence of reflection of crystalline $\mathrm{TiO}_{2}$ at low titanium loading is due to the higher dispersion of titanium within the silica matrix, which can prevent the formation of $\mathrm{TiO}_{2}$ domains sufficiently large to be detected by XRD.

Figure 2 shows the UV-vis diffuse reflectance spectra of the prepared samples to give the existence state of the titanium. The sample absorption peaks have an obvious blue shift with decreasing of content of titanium, caused by quantum size effect of semiconductor compound [16]. Ti-SBA-15 exhibits a typical absorption peak at $210 \mathrm{~nm}$, which can be assigned to the ligand to metal charge transfer transition of tetrahedrally coordinated Ti-oxide moieties [17, 18]. The absorption bands near $330 \mathrm{~nm}$ were attributed to the formation of $\mathrm{TiO}_{2}$ clusters in the anatase form when the $\mathrm{Si} / \mathrm{Ti}$ ratio is 10 [19].

The FT-IR spectra of the titanium incorporated and hydrothermally prepared mesoporous molecular sieves are presented in Figure 3. For Ti-SBA-15 samples, the IR absorption at about $960 \mathrm{~cm}^{-1}$ usually is characteristic of the Ti-O$\mathrm{Si}$ vibration. However, there are still some deputes over the assignment of the band at $960 \mathrm{~cm}^{-1}$ in titanium-containing molecular sieves, which is often assigned to asymmetric $\mathrm{Si}-$ $\mathrm{O}-\mathrm{Ti}$ vibration and correlated with the presence of $\mathrm{Si}-\mathrm{OH}$ group [20]. In the present work, the band at about $960 \mathrm{~cm}^{-1}$ is slightly weak in pure siliceous SBA-15. With increasing the titanium content, the $960 \mathrm{~cm}^{-1}$ band is enhanced whereas the band at $800 \mathrm{~cm}^{-1}$ corresponding to the $\mathrm{Si}-\mathrm{O}-\mathrm{Si}$ vibration decreases. This result suggests that systematic increase in intensity of FT-IR absorption with increasing the titanium amount is the proof of $\mathrm{Ti}$ incorporating into the framework of SBA-15 [11].

Figures 4(a) and 4(b) shows O1s and Ti2p XPS spectra of Ti-SBA-15 samples with various titanium loadings. The O1s XPS peak is very broad and asymmetric for a single band (Figure 4(a)). The main feature at $535.0 \mathrm{eV}$ is unambiguously assigned to oxygen atoms bonded to silicon, which is consistent with earlier report [21]. A peak at an intermediate BE value of $531.6 \mathrm{eV}$ is clearly presented, which is assigned to oxygen in interfacial $\mathrm{Si}-\mathrm{O}-\mathrm{Ti}$ cross-linking bonds, showing titanium was incorporated in the framework of support [22]. However, another peak at $528.4 \mathrm{eV}$ can be seen on Ti-SBA15 samples $(\mathrm{Si} / \mathrm{Ti}=10)$ and its intensity was increased with increasing of the titanium loadings. The possible reason is that the mixing anatase and rutile titanium species in

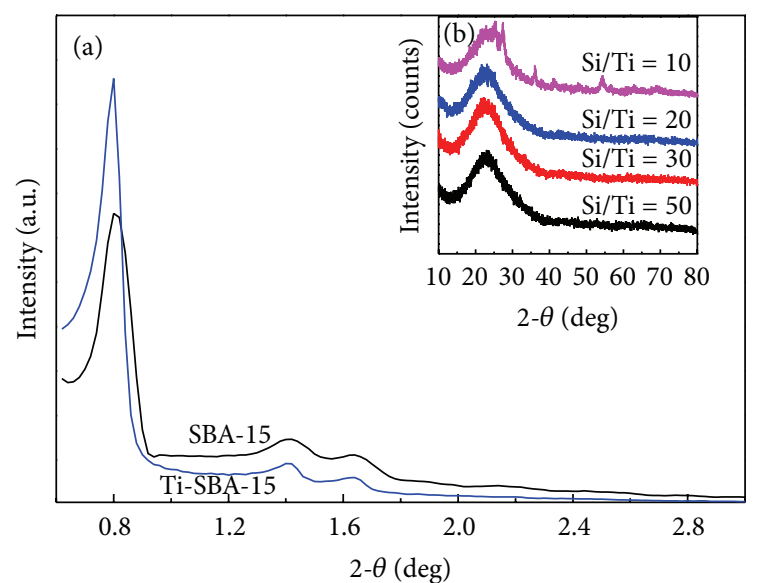

FIGURE 1: (a) Low-angle XRD patterns of sample SBA-15 and (b) Ti-SBA-15 $(\mathrm{Si} / \mathrm{Ti}=10)$ and high-angel XRD patterns of different Ticontaining materials.

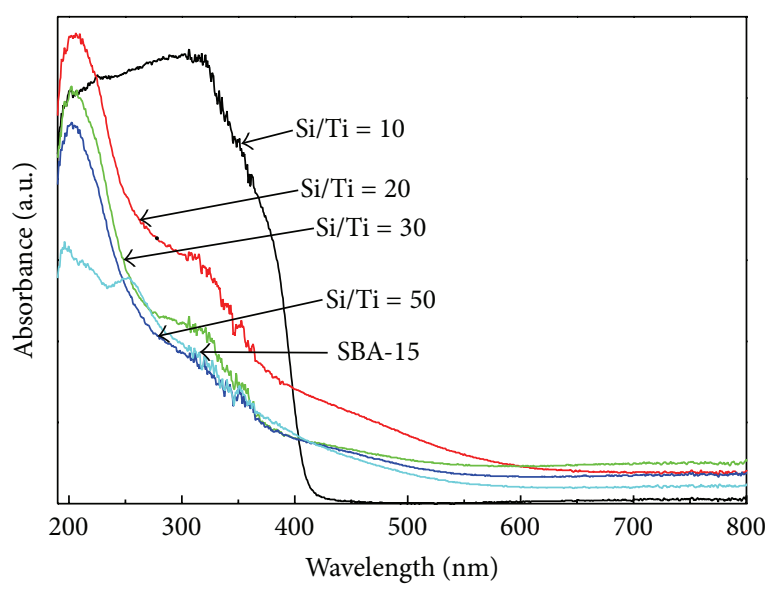

FIgURE 2: DRS UV-Vis spectra of different Ti-containing samples.

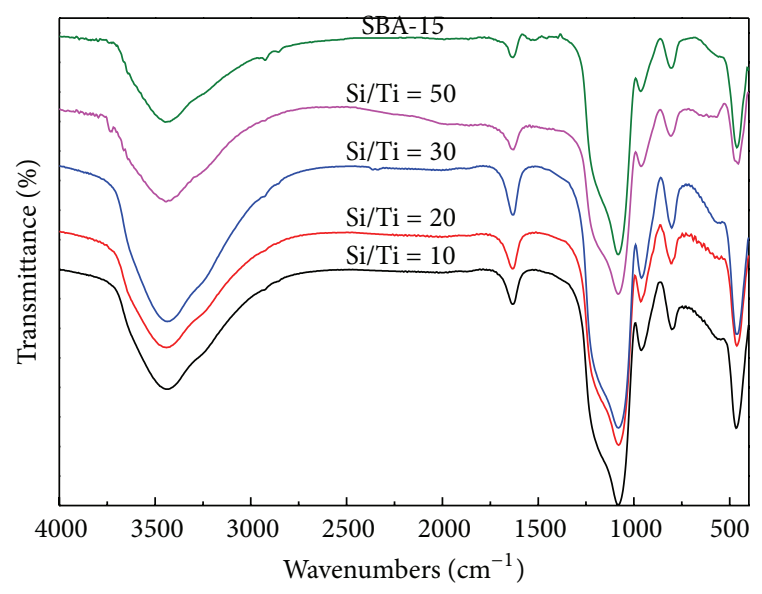

FIGURE 3: FT-IR spectra of different Ti-containing samples. 


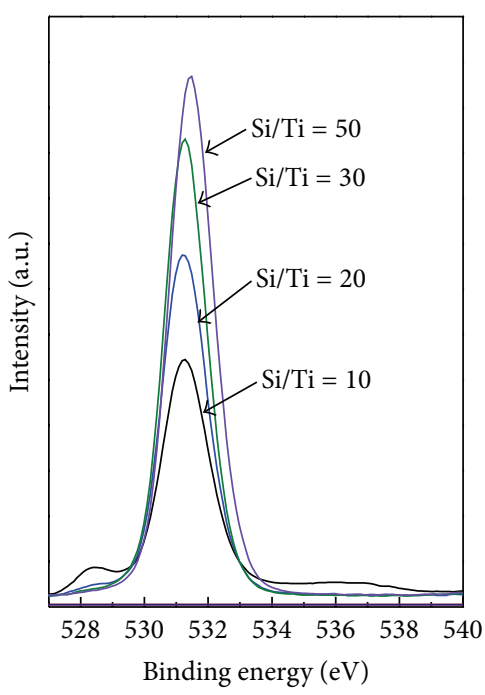

(a)

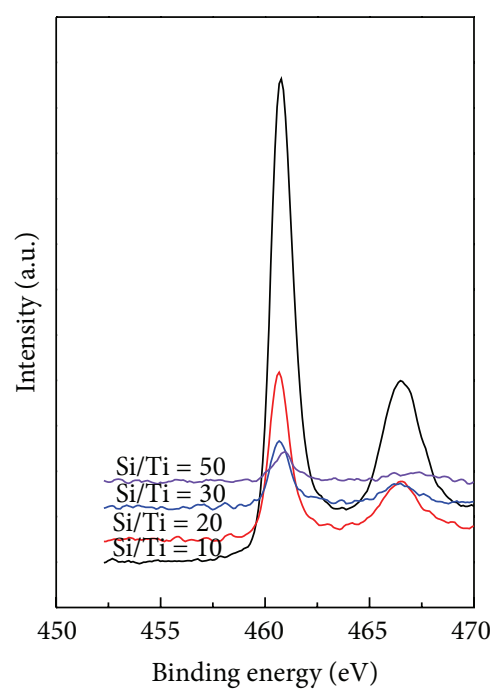

(b)

FIGURE 4: XPS spectra of different titanium content of Ti-SBA-15: (a) O1s core-level spectra and (b) Ti2p core-level spectra.

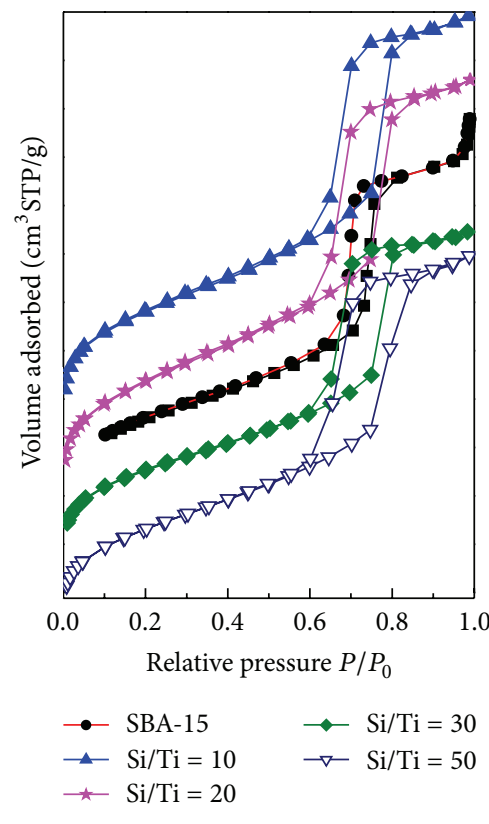

(a)

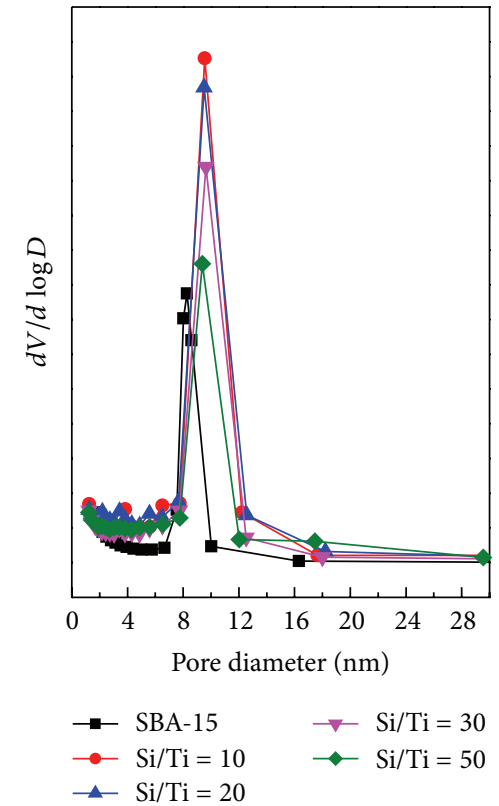

(b)

FIGURE 5: Nitrogen adsorption-desorption isotherms (a) and pore size distribution curve of different titanium content of Ti-SBA-15 (b).

the Ti-SBA-15 were formed at higher loadings [23], which are not detected in high angle XRD. The Ti2p XPS spectra was given in Figure 4(b). The largest central peak (at $460.3 \mathrm{eV}$ and $466 \mathrm{eV}$ ) was assigned to cross-linking Ti-O-Si bonds. Based on the above results combined with UV-Vis, and FTIR spectra, it can be concluded that titanium ions were successfully incorporated into the framework of SBA-15.

The $\mathrm{N}_{2}$ adsorption-desorption measurement was performed to elucidate the changes in the surface area, pore size distribution as well as pore volume. Figure 5(a) shows the $\mathrm{N}_{2}$ adsorption-desorption isotherm of each sample at
$77 \mathrm{~K}$. The typical type IV isotherm was observed with large type $\mathrm{H} 1$ hysteresis loops at high relative pressure $\left(P / P_{0}=\right.$ $0.6-0.8)$. The sharpness of steps also displays a typical ordered mesoporous material prepared with larger pore sizes and narrow size distribution [14]. These are in accordance with those measured from the low angle XRD patterns.

The pore size distribution curve of each sample was also shown in Figure 5(b), and the results from the analysis of these isotherms were summarized in Table 1. The BJH pore size distribution curves reveal an average pore size of about $9 \mathrm{~nm}$ for the Ti-SBA-15 samples, which is larger than 
TABLE 1: Pore structure character parameters of SBA-15 and Ti-SBA15.

\begin{tabular}{lccc}
\hline Samples & $\begin{array}{c}\text { BET area } \\
\left(\mathrm{m}^{2} / \mathrm{g}\right)\end{array}$ & $\begin{array}{c}\text { Mesopore volume }^{\mathrm{a}} \\
\left(\mathrm{cm}^{3} / \mathrm{g}\right)\end{array}$ & $\begin{array}{c}\text { Pore size }^{\mathrm{b}} \\
(\mathrm{nm})\end{array}$ \\
\hline SBA-15 & 938 & 1.29 & 6.64 \\
Ti-SBA-15 $(\mathrm{Si} / \mathrm{Ti}=10)$ & 1091 & 1.38 & 9.54 \\
Ti-SBA-15 $(\mathrm{Si} / \mathrm{Ti}=20)$ & 1156 & 1.42 & 9.50 \\
Ti-SBA-15 $(\mathrm{Si} / \mathrm{Ti}=30)$ & 978 & 1.26 & 9.23 \\
Ti-SBA-15 $(\mathrm{Si} / \mathrm{Ti}=50)$ & 913 & 1.12 & 9.24 \\
\hline
\end{tabular}

${ }^{\mathrm{a}} \mathrm{BJH}$ method cumulative adsorption pore volume.

${ }^{\mathrm{b}} \mathrm{BJH}$ adsorption average pore diameter.

that of the pure silica SBA-15 sample $(6.64 \mathrm{~nm})$. The surface area of Ti-containing samples is larger than SBA-15. Similar results were obtained in previous literature [24]. The obvious increase in the mesopore size upon Ti substituted samples may be due to the decrease in pore wall thickness of the titanium-containing samples. We can also find the pore size is independent of the content of titanium introduced into the samples, indicating that no titania formed on the internal surface of the mesoporous samples. Therefore, titania species could exist in the framework and/or on the external surface of SBA-15.

3.2. Photocatalytic Process of Phenol. The photocatalytic degradation efficiencies of a series of hydrothermally synthesized Ti-SBA-15 materials were performed by using phenol as a model pollutant with $100 \mathrm{mg} / \mathrm{L}$ initial concentration. Figure 6 shows the influence of the titanium content in the TiSBA-15 materials on the phenol degradation versus the time. At the same time, the more titanium the Ti-SBA-15 contains, the faster the phenol decomposes. Consequently, the TiSBA- 15 materials with $\mathrm{Si} / \mathrm{Ti}=10$ has the best photocatalytic efficiency with the degradation of phenol up to $97.2 \%$ at $120 \mathrm{~min}$. As compared, the catalytic activity of bulk $\mathrm{TiO}_{2}$ synthesized by means of hydrothermal method is shown in Figure 6. The performance of framework Ti-SBA-15 catalysts to degrade phenol is found to be better in general than that of the bulk $\mathrm{TiO}_{2}$. In addition, the activity of $\mathrm{TiO}_{2}$ deposited directly into pre-synthesized SBA- 15 with $\mathrm{Si} / \mathrm{Ti}=10$, denoted as $\mathrm{d}-\mathrm{TiO}_{2} / \mathrm{SBA}-15$, is also tested. The $\mathrm{d}-\mathrm{TiO}_{2} / \mathrm{SBA}-15$ exhibits less photodegradation ability towards phenol even compared to the bulk $\mathrm{TiO}_{2}$. This result indicates that both the high special surface and the framework titanium incorporated into SBA-15 play an important role on the decomposition of phenol and acts as photocatalytic active sites. It can be explained that the higher dispersion in Ti-SBA-15 provides more surface sites for the adsorption of reactants molecular and makes the process more efficient.

It is well known that hydrogen peroxide with ultraviolet radiation can be used for the photodegradation of pollutants in homogenous and heterogeneous systems. In order to further ascertain the effect of hydrogen peroxide and Ti-SBA-15 $(\mathrm{Si} / \mathrm{Ti}=20)$, Figure 7 shows photocatalytic decomposition of phenol based on different combination of hydrogen peroxide, Ti-SBA-15 and ultraviolet radiation. Experimental results

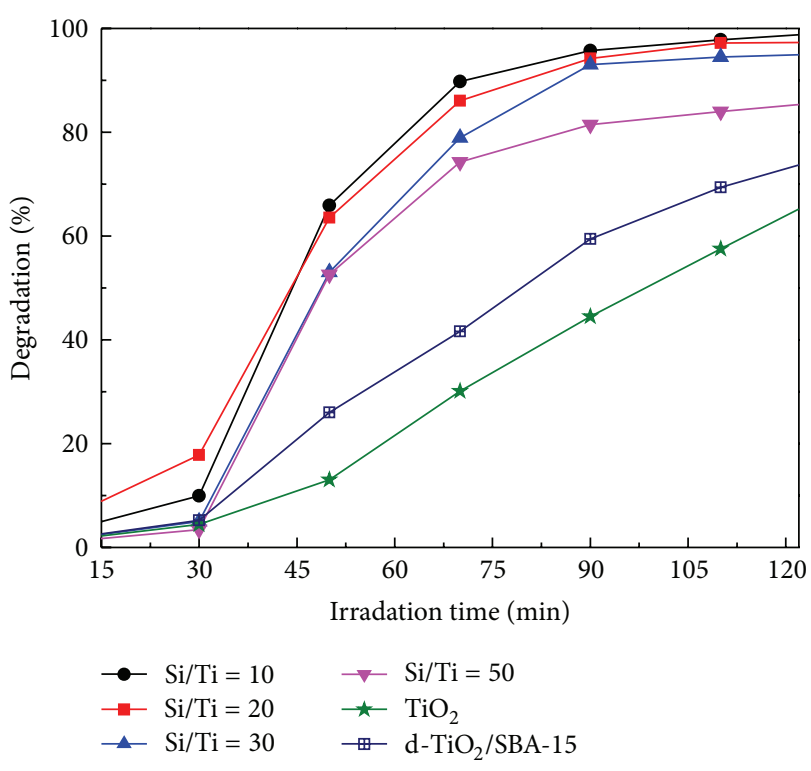

FIgURE 6: Photocatalytic degradation of Ti-SBA-15 to phenol under different $\mathrm{Si} / \mathrm{Ti}$ ratio. $\mathrm{d}-\mathrm{TiO}_{2} / \mathrm{SBA}-15$ is $\mathrm{TiO}_{2}$ deposited directly into pre-synthesized SBA-15.

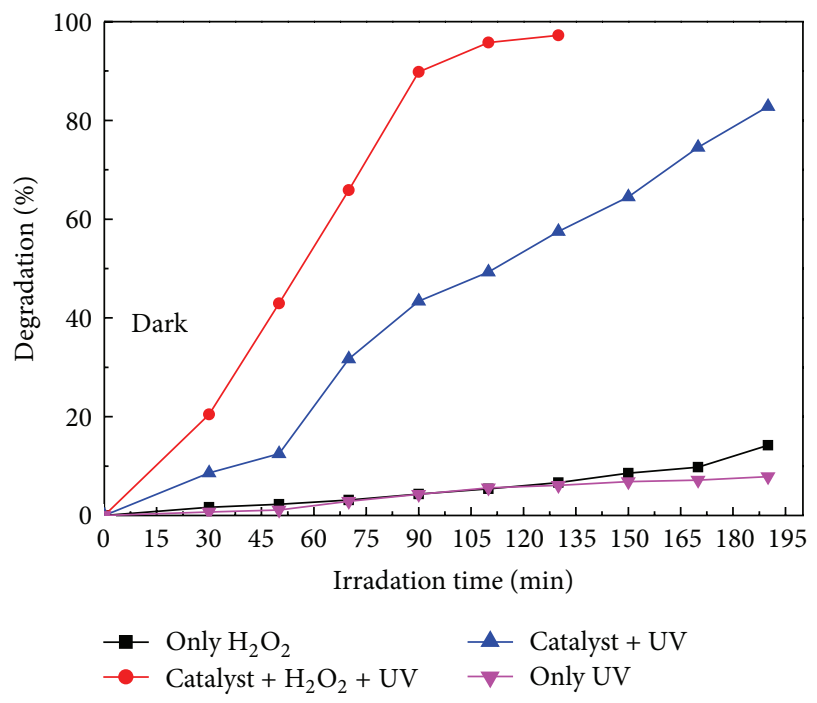

Figure 7: Phenol photodegradation route with different process combination.

indicated that the addition of hydrogen peroxide benefited to the efficiency of photodegradation towards phenol in the presence of Ti-SBA-15. The degradation of phenol is $20 \%$ less than that only with hydrogen peroxide or ultraviolet radiation under $120 \mathrm{~min}$. However, the degradation of phenol increases to $82.7 \%$ with the combination of ultraviolet radiation and Ti-SBA-15. The results above suggest that both the ultraviolet radiation and Ti-SBA-15 are important for the degradation of phenol. Moreover, the addition of hydrogen peroxide can improve the degradation of phenol up to $97.2 \%$. Several studies have proposed different reaction mechanisms for the photolysis of organics in the presence of hydrogen peroxide. 
And the main interactions of hydrogen peroxide with free radicals and UV radiations are represented by the reactions listed below [12]:

$$
\begin{aligned}
& \text { Initiation: } \mathrm{H}_{2} \mathrm{O}_{2}+\sum h v \longrightarrow 2 \cdot \mathrm{OH} \\
& \text { Propagation: } \mathrm{H}_{2} \mathrm{O}_{2}+\bullet \mathrm{OH} \longrightarrow \cdot \mathrm{HO}_{2}+\mathrm{H}_{2} \mathrm{O} \\
& \mathrm{H}_{2} \mathrm{O}_{2}+\cdot \mathrm{HO}_{2} \longrightarrow \cdot \mathrm{OH}+\mathrm{H}_{2} \mathrm{O}+\mathrm{O}_{2} \\
& \text { Decomposition: } \mathrm{RH}+\bullet \mathrm{OH} \longrightarrow \text { Products } \\
& \mathrm{RH}+\cdot \mathrm{HO}_{2} \longrightarrow \text { Products } \\
& \text { Termination: } 2 \cdot \mathrm{OH} \longrightarrow \mathrm{H}_{2} \mathrm{O}_{2} \\
& \text { - } \mathrm{HO}_{2} \longrightarrow \mathrm{H}_{2} \mathrm{O}_{2}+\cdot \mathrm{O}_{2}{ }^{-} \\
& \cdot \mathrm{OH}+\cdot \mathrm{HO}_{2} \longrightarrow \mathrm{H}_{2} \mathrm{O}+\mathrm{O}_{2}
\end{aligned}
$$

where RH means organic to be decomposed (Here is specially denoted as phenol). The degradation begins with the formation of electron-hole pairs in the Ti-SBA-15 upon absorption of irradiation, followed by the generation of different reactive oxygen species, which induce the degradation of the organic compounds. Figure 8 presented the influence of concentration of hydrogen peroxide on the degradation of phenol. The direct photolysis of hydrogen peroxide can generate free radicals, as cited in the earlier studies of photooxidation using $\mathrm{UV} / \mathrm{H}_{2} \mathrm{O}_{2}$. It causes rate-improving of photodegradation according to the decomposition reactions. However, when the content of hydrogen peroxide increases, the excess hydrogen peroxide will scavenge the $\mathrm{HO}$ - generated by the direct photolysis of hydrogen peroxide or the photooxidation of $\mathrm{HO} \cdot$ by electron-hole pairs, leading to the much weaker radical of $\bullet \mathrm{HO}_{2}$ forms. Thus, the total oxidation properties of the system are reduced by termination reaction. The effect of hydrogen peroxide on phenol photodecomposition can be explained by the oxidative action of radical species produced from oxygen photoreduction and hydrogen peroxide photodecomposition on titanium dioxide surface. However, suitable concentration of hydrogen peroxide, prohibiting the electron-hole recombination, could act as an alternative electron acceptor to oxygen, in that hydrogen peroxide is a better electron acceptor than molecular oxygen. Experiments have demonstrated the beneficial effect of the addition of hydrogen peroxide on the efficiency of phenol photodegradation in the presence of Ti-SBA-15.

The Ti-SBA-15 as heterogenous catalysts in this study can be easily recovered via simple filtration, followed by rinsing with water, drying and calcinating at $400^{\circ} \mathrm{C}$ for $4 \mathrm{~h}$. The reusability of Ti-SBA-15 was also examined, and the results are shown in Figure 9. The stability of the photocatalytic activity was assessed at a constant initial concentration of phenol $(100 \mathrm{mg} / \mathrm{L}$ and natural $\mathrm{pH})$. The recycling catalysts were found to maintain catalytic activities with little loss of activity after six runs. The results show that the photocatalyst is basically stable and has good prospects for treating wastewater containing phenol.

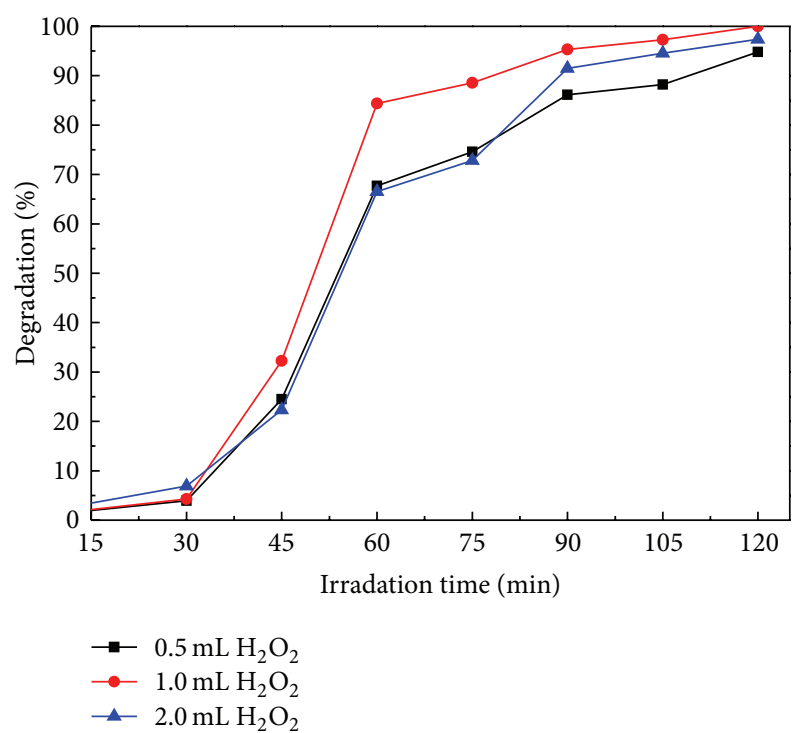

FIGURE 8: The influence of hydrogen peroxide concentration on the degradation of phenol.

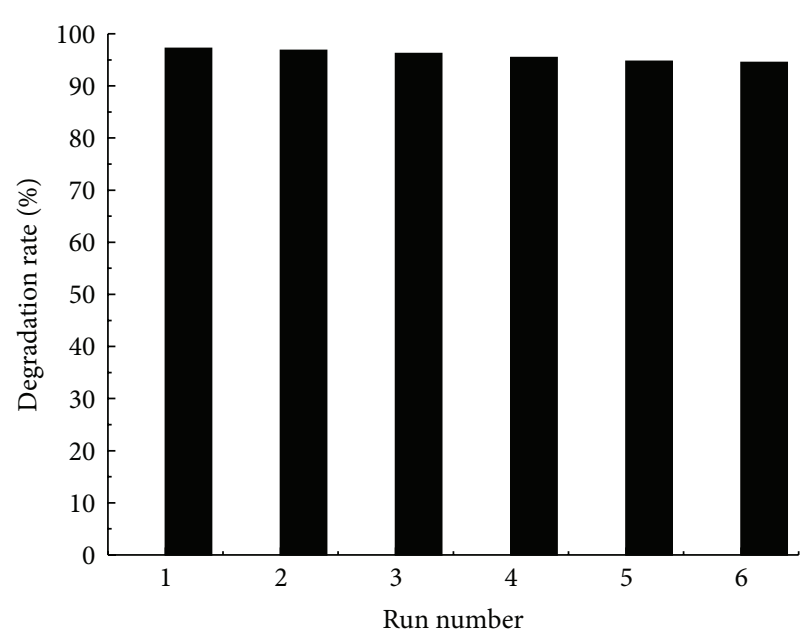

Figure 9: Reusability of the Ti-SBA-15 $(\mathrm{Si} / \mathrm{Ti}=20)$ catalyst toward the degradation of phenol in solution.

\section{Conclusions}

In our study, the Ti-containing SBA-15 materials were synthesized by one-pot hydrothermal method, and titanium could be effectively incorporated into the framework of support and mainly existed in tetrahedrally coordinated Tioxide according to a series of characterization. The catalysts were applied to degrade phenol solution to evaluate their photocatalytic activity. The results indicate photocatalytic degradation is an effective method for completely removal of phenol with the assistance of hydrogen peroxide, and the more titanium content and anatase titanium dioxide are responsible for the high photocatalytic activity. The used catalysts could be easily regenerated by calcinating at $400^{\circ} \mathrm{C}$ for $4 \mathrm{~h}$, and were found to maintain catalytic activities after recycling. 


\section{Conflict of Interests}

The authors declare that there is no conflict of interests regarding the publication of this paper.

\section{Acknowledgments}

The authors thank the China-Japan International Cooperation Project (2013DFG50150), the Natural Science Foundation of China (no. 21476267) and Japan Society for the Promotion of Science for supporting this work.

\section{References}

[1] S. S. Adav, M.-Y. Chen, D.-J. Lee, and N.-Q. Ren, "Degradation of phenol by Acinetobacter strain isolated from aerobic granules," Chemosphere, vol. 67, no. 8, pp. 1566-1572, 2007.

[2] G. Busca, S. Berardinelli, C. Resini, and L. Arrighi, "Technologies for the removal of phenol from fluid streams: a short review of recent developments," Journal of Hazardous Materials, vol. 160, no. 2-3, pp. 265-288, 2008.

[3] S. Ahmed, M. G. Rasul, W. N. Martens, R. Brown, and M. A. Hashib, "Heterogeneous photocatalytic degradation of phenols in wastewater: a review on current status and developments," Desalination, vol. 261, no. 1-2, pp. 3-18, 2010.

[4] A. Kumar and D. Srinivas, "Hydroxylation of phenol with hydrogen peroxide catalyzed by Ti-SBA-12 and Ti-SBA-16," Journal of Molecular Catalysis A Chemical, vol. 368-369, pp.112$118,2013$.

[5] P. P. Hankare, R. P. Patil, A. V. Jadhav, K. M. Garadkar, and R. Sasikala, "Enhanced photocatalytic degradation of methyl red and thymol blue using titania-alumina-zinc ferrite nanocomposite," Applied Catalysis B: Environmental, vol. 107, no. 3-4, pp. 333-339, 2011.

[6] V. Durgakumari, M. Subrahmanyam, K. V. S. Rao, A. Ratnamala, M. Noorjahan, and K. Tanaka, "An easy and efficient use of $\mathrm{TiO}_{2}$ supported HZSM-5 and $\mathrm{TiO}_{2}+\mathrm{HZSM}-5$ zeolite combinate in the photodegradation of aqueous phenol and $\mathrm{p}$ chlorophenol," Applied Catalysis A: General, vol. 234, no. 1-2, pp. 155-165, 2002.

[7] Y. A. Shaban, M. A. El Sayed, A. A. El Maradny, R. K. Al Farawati, and M. I. Al Zobidi, "Photocatalytic degradation of phenol in natural seawater using visible light active carbon modified (CM)-n- $\mathrm{TiO}_{2}$ nanoparticles under UV light and natural sunlight illuminations," Chemosphere, vol. 91, no. 3, pp. 307-313, 2013.

[8] P. Yang, D. Zhao, D. I. Margolese, B. F. Chmelka, and G. D. Stucky, "Generalized syntheses of large-pore mesoporous metal oxides with semicrystalline frameworks," Nature, vol. 396, no. 6707, pp. 152-155, 1998.

[9] G. Liu, Y. Liu, G. Yang et al., "Preparation of titania-silica mixed oxides by a sol-gel route in the presence of citric acid," Journal of Physical Chemistry C, vol. 113, no. 21, pp. 9345-9351, 2009.

[10] S. Perathoner, P. Lanzafame, R. Passalacqua, G. Centi, R. Schlögl, and D. S. Su, "Use of mesoporous SBA-15 for nanostructuring titania for photocatalytic applications," Microporous and Mesoporous Materials, vol. 90, no. 1-3, pp. 347-361, 2006.

[11] W. Y. Jung, S. H. Baek, J. S. Yang et al., "Synthesis of Ticontaining SBA-15 materials and studies on their photocatalytic decomposition of orange II," Catalysis Today, vol. 131, no. 1-4, pp. 437-443, 2008.
[12] W. Song, V. Ravindran, and M. Pirbazari, "Process optimization using a kinetic model for the ultraviolet radiation-hydrogen peroxide decomposition of natural and synthetic organic compounds in groundwater," Chemical Engineering Science, vol. 63, no. 12, pp. 3249-3270, 2008.

[13] B. L. Newalkar, J. Olanrewaju, and S. Komarneni, "Direct synthesis of titanium-substituted mesoporous SBA-15 molecular sieve under microwave - Hydrothermal conditions," Chemistry of Materials, vol. 13, no. 2, pp. 552-557, 2001.

[14] D. Zhao, J. Feng, Q. Huo et al., "Triblock copolymer syntheses of mesoporous silica with periodic 50 to 300 angstrom pores," Science, vol. 279, no. 5350, pp. 548-552, 1998.

[15] D. Cani and P. P. Pescarmona, "Macroscopic TiO2-SiO2 porous beads: Efficient photocatalysts with enhanced reusability for the degradation of pollutants," Journal of Catalysis, vol. 311, pp. 404411, 2014.

[16] M. A. Henderson, "A surface science perspective on $\mathrm{TiO}_{2}$ photocatalysis," Surface Science Reports, vol. 66, no. 6-7, pp. 185297, 2011.

[17] F. Bérubé, F. Kleitz, and S. Kaliaguine, "Surface properties and epoxidation catalytic activity of Ti-SBA15 prepared by direct synthesis," Journal of Materials Science, vol. 44, no. 24, pp. 6727$6735,2009$.

[18] T. Kamegawa, N. Suzuki, K. Tsuji et al., "Preparation of hydrophobically modified single-site Ti-containing mesoporous silica (TiSBA-15) and their enhanced catalytic performances," Catalysis Today, vol. 175, no. 1, pp. 393-397, 2011.

[19] J. A. Melero, J. M. Arsuaga, P. D. Frutos, J. Iglesias, J. Sainz, and S. Blázquez, "Direct synthesis of titanium-substituted mesostructured materials using non-ionic surfactants and titanocene dichloride," Microporous and Mesoporous Materials, vol. 86, no. 1-3, pp. 364-373, 2005.

[20] P. V. Suraja, Z. Yaakob, N. N. Binitha, M. R. Resmi, and P. P. Silija, "Photocatalytic degradation of dye pollutant over Ti and Co doped SBA-15: comparison of activities under visible light," Chemical Engineering Journal, vol. 176-177, pp. 265-271, 2011.

[21] B. J. Aronson, C. F. Blanford, and A. Stein, "Solution-phase grafting of titanium dioxide onto the pore surface of mesoporous silicates: synthesis and structural characterization," Chemistry of Materials, vol. 9, no. 12, pp. 2842-2851, 1997.

[22] M. Mohai, I. Bertoti, and M. Revesz, "XPS study of the state of oxygen on a chemically treated glass surface," Surface and Interface Analysis, vol. 15, no. 6, pp. 364-368, 1990.

[23] G. Li and X. S. Zhao, "Characterization and photocatalytic properties of titanium-containing mesoporous SBA-15," Industrial \& Engineering Chemistry Research, vol. 45, no. 10, pp. 3569-3573, 2006.

[24] C.-H. Huang, K.-P. Chang, H.-D. Ou, Y.-C. Chiang, E.-E. Chang, and C.-F. Wang, "Characterization and application of Ti-containing mesoporous silica for dye removal with synergistic effect of coupled adsorption and photocatalytic oxidation," Journal of Hazardous Materials, vol. 186, no. 2-3, pp. 1174-1182, 2011. 

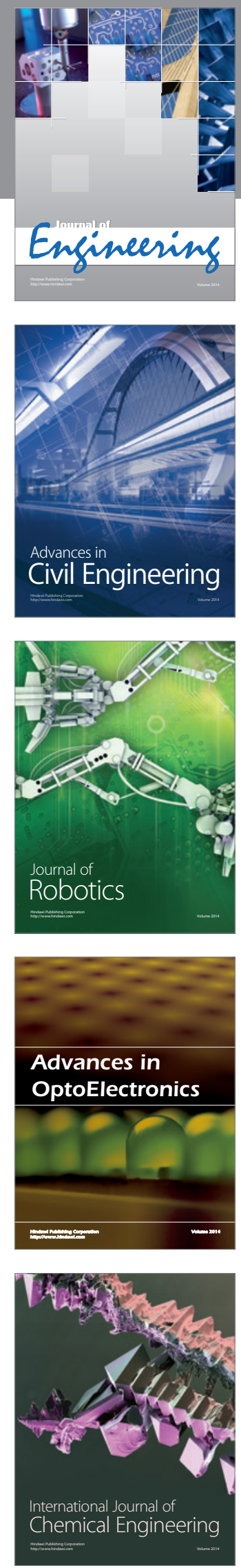

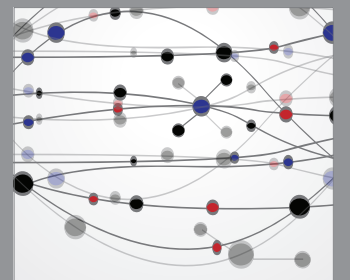

The Scientific World Journal
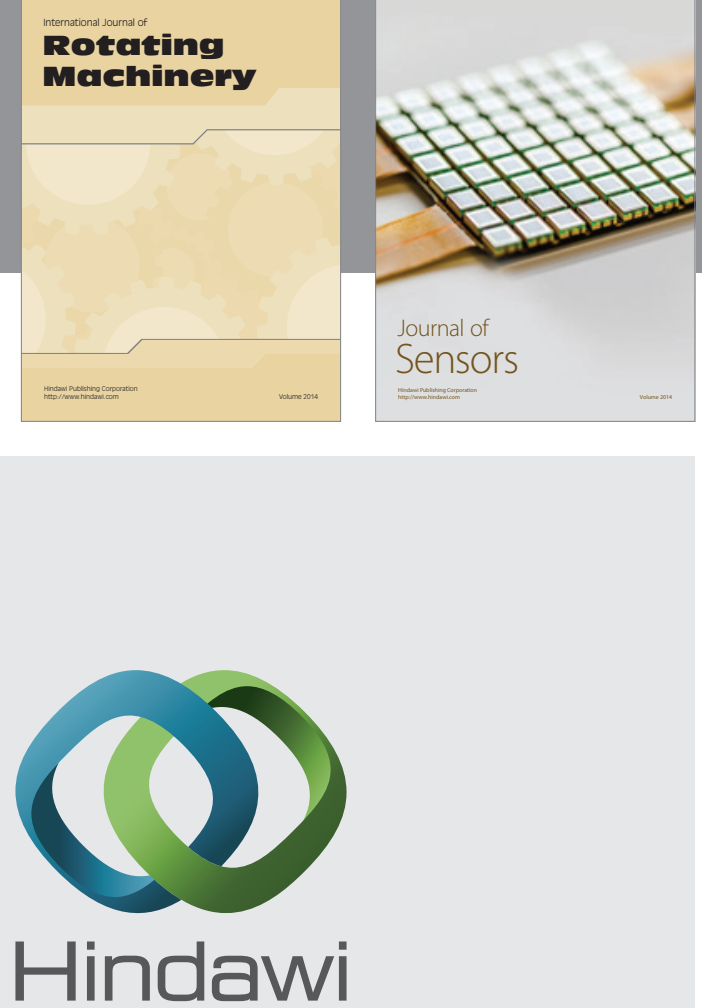

Submit your manuscripts at http://www.hindawi.com
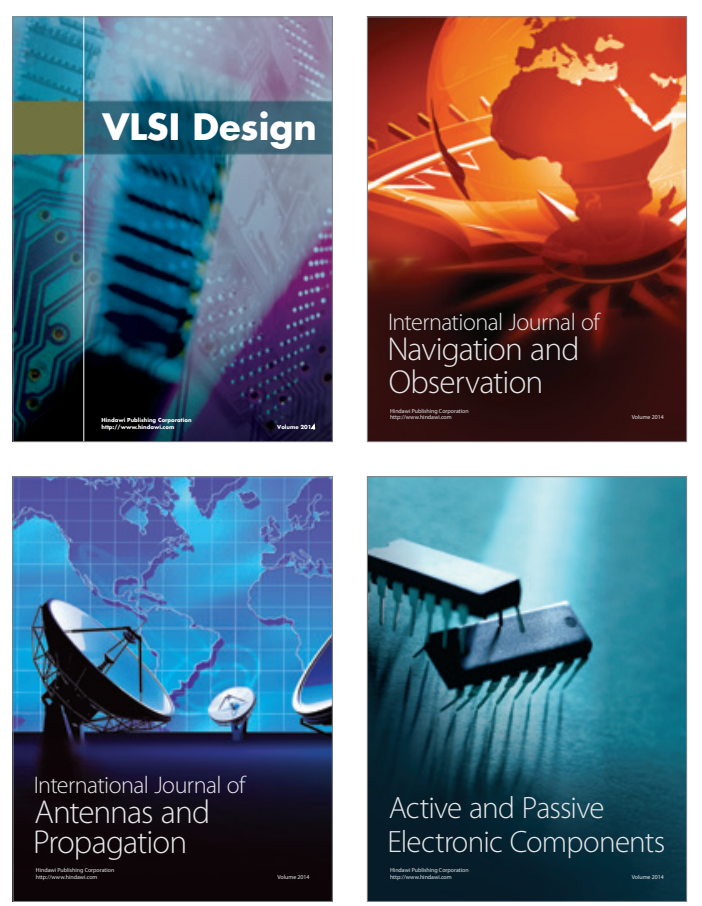
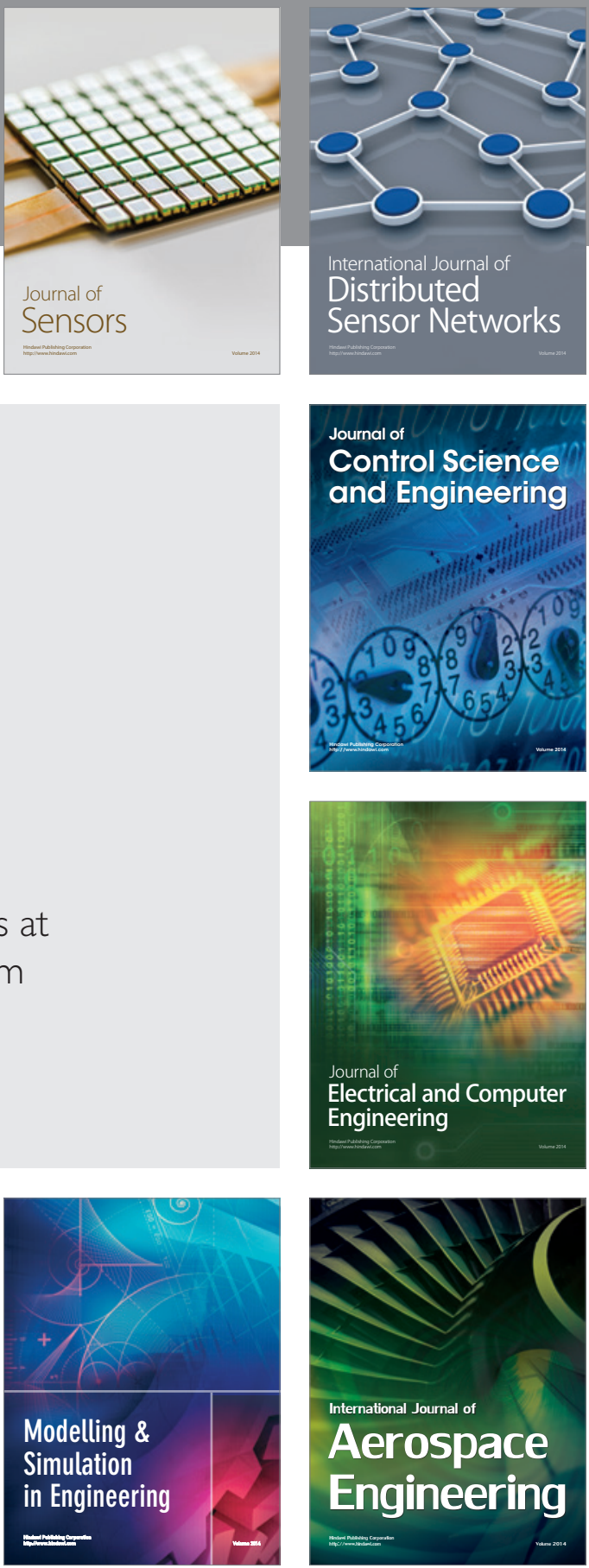

Journal of

Control Science

and Engineering
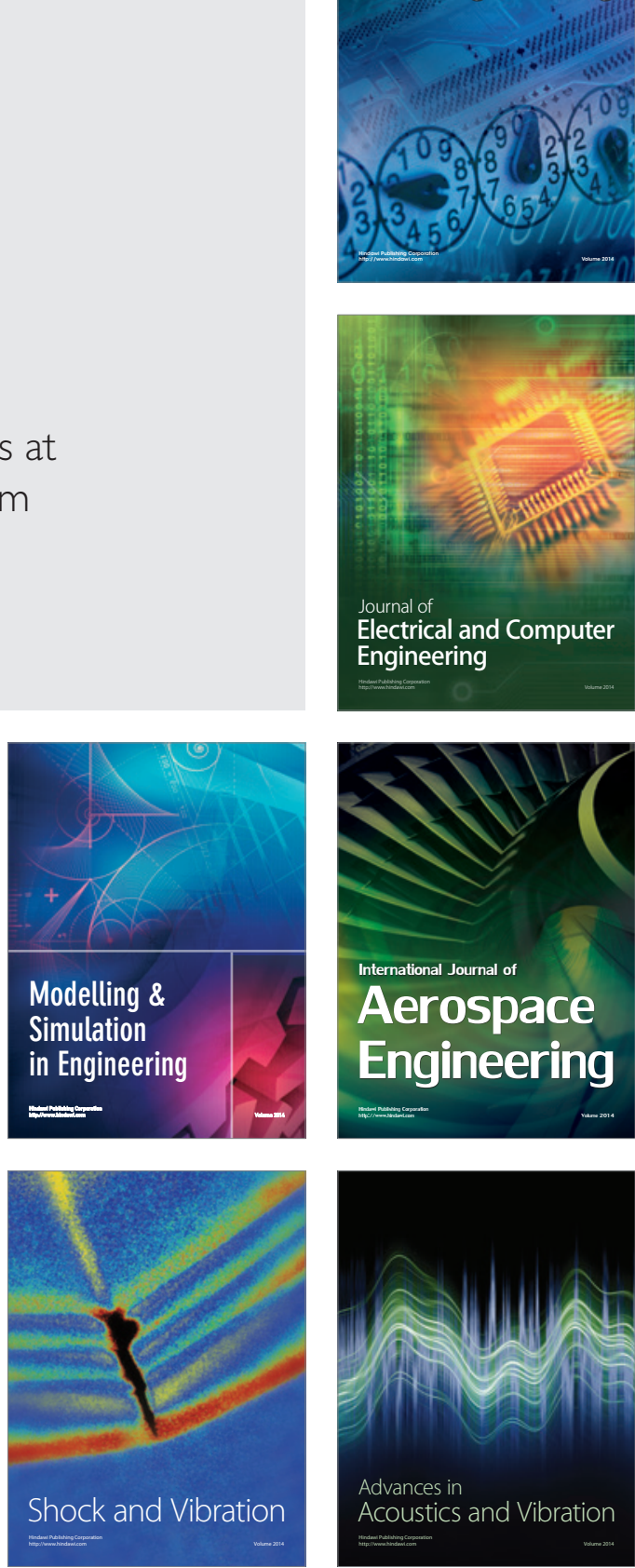